\title{
Dyslipidaemia: what's around the corner?
}

\author{
Authors: Anthony S Wierzbicki, ${ }^{A}$ Dilinika Perera ${ }^{\mathrm{B}}$ and Mfon Ewang-Emukowhate ${ }^{\mathrm{C}}$
}

Hyperlipidaemia is a major risk factor for the development of atherosclerosis and cardiovascular disease. Statins are the mainstay of therapy and new guidelines focus on the use of these agents without specific targets for low-density lipoprotein (LDL)-cholesterol or non high-density lipoprotein (HDL)cholesterol. However, patients remain at risk of cardiovascular disease despite statin therapy so new drugs are required. This article reviews therapies in development to further lower LDLcholesterol (Proprotein convertase subtilisin/kexin-9 (PCSK-9) inhibitors), raise HDL-holesterol (cholesterol ester transfer protein inhibitors (CETPIs)) and reduce triglycerides (novel peroxisome proliferator-activated receptor (PPAR)-agonists and omega-3 fatty acid preparations). Specialised therapies are in development for treatment of orphan disoders such as homozygous familial hypercholesterolaemia (lomitapide) or familial chylomicroaemia (alipogene tiparvovec). These novel lipid-lowering agents are likely to find uses in treating patients at the highest cardiovascular risk.

KEYWORDS: Cardiovascular disease, hyperlipidaemia, cholesterol, PCSK9, cholesterol ester transfer protein inhibitor

\section{Introduction}

Atherosclerosis and its consequent cardiovascular disease (CVD) is one of the most common causes of morbidity and mortality worldwide. Its incidence is increasing globally as nutrition improves, western lifestyles are adopted and populations age. Hyperlipidaemia, or more accurately dyslipidaemia, is a major risk factor for CVD and may account for up to $55 \%$ of age and gender-independent risk. ${ }^{1}$ The dyslipidaemia component that accounts for the epidemiological risk is the ratio of total cholesterol to highdensity lipoprotein-cholesterol (HDL-C), or alternatively the ratio of apolipoprotein B:A-1 concentrations, which identifies the fraction of lipid particles (whether triglyceride-rich or not) depositing cholesterol in the vascular wall compared to those removing it. At its simplest, this process can be expressed as non-HDL-C (difference of total and HDL-C) vs HDL-C.

Dyslipidaemia had been treated with varying degrees of enthusiasm for 50 years but has become one of the major

Authors: Aconsultant, Guy's and St Thomas' Hospitals, London, UK; Bspecialist registrar, Guy's and St Thomas' Hospitals, London, UK;

'specialist registrar, Guy's and St Thomas' Hospitals, London, UK treatment priorities since the publication of the Scandinavian Simvastatin Survival Study in $1994,{ }^{2}$ which showed that statins not only decreased CVD but also reduced mortality in high-risk patients with established coronary heart disease (CHD). Later statin studies have extended the indications for this treatment to include stroke, CVD in diabetes and the treatment of asymptomatic but higher risk individuals in primary prevention. ${ }^{3}$ Analyses of surrogate outcome measures such as intravascular ultrasound measured atheroma volume and epidemiological studies suggest that, though CVD risk is exponential when plotted against low-density lipoproteincholesterol (LDL-C) or non-HDL-C concentrations, significant risk only begins to accrue above an LDL-C of $2 \mathrm{mmol} / \mathrm{l}$ or non-HDL-C of $2.8 \mathrm{mmol} / \mathrm{l}$. The benefits of statins can be summarised as a $20 \%$ reduction in relative risk of CVD events per $1 \mathrm{mmol} / \mathrm{l}$ reduction in LDL-C, if a linear model is assumed. ${ }^{3}$

\section{The guidelines debate}

Recent guidelines from the USA ${ }^{4}$ and UK (draft NICE guidelines $)^{5}$ have incorporated the evidence from statin trials into clinical practice and, noting the far lesser benefits of other treatments (fibrates, niacin and omega-3 fatty acids), have suggested maximising statin therapy in patients with CVD or its risk equivalents (eg diabetes and chronic kidney disease stage 3). These guidelines have abandoned targets for LDL-C based on fundamentals of trial designs and the lack of evidence for add-on therapies. In contrast, the European societies' guidelines retain targets for LDL-C of $2.5 \mathrm{mmol} / \mathrm{l}$ and preferably $<2 \mathrm{mmol} / \mathrm{l}$ in patients with CVD. ${ }^{6}$ In essence this difference relates to two different views of the benefits of lipid-lowering and how they should be assessed. In one view, outcomes evidence trumps all other surrogate measures; holders of this view note that some statin trials (eg in grade $2 / 3$ heart failure or chronic kidney disease (CKD)3-5) have been unsuccessful despite large reductions in LDL-C. The other view is that CKD3-5 and heart failure represent conditions where atherothrombotic events are rare and atherosclerosis is too prevalent and well established to respond to lipid-lowering therapy in short-term trials, and that reductions in LDL-C will generally lead to reductions in CVD however they are achieved. As few trials or interventions have addressed HDL-C or triglycerides, these are generally viewed by guidelines as modifiers of underlying risk or determinants of residual risk after LDL-C has been addressed. Many compounds are in development, given the importance of lipids to CVD risk. ${ }^{7,8}$ 


\section{New lipid-lowering drugs for LDL-cholesterol}

Statins are generally well tolerated but increased rates of myalgia and myositis are known common problems with these drugs. The frequency of these side-effects is disputed, ranging from minimal in the placebo-controlled trials to reports of rates up to $15 \%$ in general use. ${ }^{9}$ Some of these problems can be addressed by switching statins or intermittent dosing regimes..$^{10}$ There are also patients who do not seem to respond well to statin therapy or in whom baseline LDL-C levels are so high that in many cases statins cannot reduce them to levels seen in the general population, for example patients with familial hypercholesterolaemia (FH). Therapies beyond statins to lower LDL-C exist. Bile acid sequestrants have a long history; some evidence in monotherapy exists from the lipid research clinics trial but is limited by low efficacy (20\% LDL-C reduction), poor tolerability and frequent drug interactions. Ezetimibe is commonly used, reduces LDL-C by $23 \%$ and is well tolerated. However, both surrogate and endpoint trials with ezetimibe have had unclear results. ${ }^{11}$ The results of the IMPROVE-IT monotherapy trial are expected in 2014 but given the small difference expected in an acute coronary syndrome (ACS) population, based on a $0.4 \mathrm{mmol} / \mathrm{l}$ difference in LDL-C, this may not answer the questions about this agent. There is thus a need for high efficacy, safe non-statin methods of reducing LDL-C.

\section{Inhibition of pro-protein convertase subtilisin/kexin 9}

Research into populations with FH identified dominant activating mutations in pro-protein convertase subtilisin/ kexin 9 (PCSK-9) as causing inhibition of LDL receptor function, resulting in high plasma LDL-C levels. By contrast, inactivating mutations reduced LDL-C by $0.6 \mathrm{mmol} / \mathrm{l}$ and were associated with decreased rates of CVD in African-American populations. ${ }^{12}$ A small number of PCSK-9 deficient homozygotes were identified and found to be clinically normal, suggesting that inhibition of this pathway had few potentially deleterious effects. This has led to the development of a number of strategies to pharmacologically inhibit PCSK-9.8,12 The furthest in development are humanised monoclonal antibodies to PCSK9. These drugs include alirocumab (REGN-727), evolocumab (AMG-145) and bococizumab (RN-316). Numerous trials of these agents have been conducted in populations with hypercholesterolaemia, $\mathrm{FH}$ and type 2 diabetes and in patients with documented intolerance to three or more statins. The results are consistent in showing a $50-65 \%$ reduction in LDL-C over and above any existing therapy or compared to placebo with minimal side-effects. One study in homozygous $\mathrm{FH}$, a condition where statins have limited, if any, efficacy shows that inhibition of PCSK-9 is partially effective in one-third of patients, giving a $20-30 \%$ reduction in LDL-C. ${ }^{13}$ Data are now available on 1,000 patient-years of trial experience, in some cases confirming that these drugs show consistent efficacy and good safety profiles. ${ }^{14}$ However, all of these agents need to be given as subcutaneous injections either fortnightly or monthly. Antibodies are made to these exogenous proteins, analogous to the case of anti-tumour necrosis factor (TNF) antibody therapies used in rheumatology, but this has not proved a problem to date. At this stage in the development of statins, both myalgia/ myositis and gastrointestinal disturbance had been identified as side-effects of statin therapy. Endpoint trials are underway with all three drugs either in populations with ACS (alirocumab; ODYSSEY outcomes) or chronic CHD (evolocumab; FOURIER; bocucizumab, SPIRE-2) and are due to report in 2016-18. One interesting feature of the design of these studies is that optimal control of LDL-C to $<2 \mathrm{mmol} / \mathrm{l}$ is assumed, so subjects recruited to these studies must have additional risk factors (eg smoking, diabetes or the metabolic syndrome) to drive event rates to significant levels.

The other approach to inhibiting PCSK-9 is to use antisense oligonucleotides (ASOs). One compound, ALN-PCS, has reached phase I human trials and after intravenous infusion reduced LDL-C by $25 \% .{ }^{15}$ Further trials are required to see if similar efficacy to the antibody-based therapies can be achieved and whether the injection site reactions characteristic of ASO therapies occur and are manageable. Attempts have been made to make small molecule inhibitors of PCSK-9 as the protein functions as an autocatalytic dimer, but these have not been successful to date. ${ }^{12}$

\section{Interventions to raise HDL-cholesterol}

Epidemiology and residual event rates within clinical trials of statins consistently show that higher levels of HDL-C are associated with better prognosis. ${ }^{16}$ However there are few interventions trials for HDL-C. Statins have little effect on HDL-C. One fibrate trial, the VA-HIT trial, showed a $25 \%$ reduction in events with gemfibrozil in a low-HDL-C population with a drug that had no effect on LDL-C. However, though outcomes correlated with an elevation in HDL-C, this effect at $6 \%$ was modest. ${ }^{17}$ Niacin raises HDL-C by $25 \%$ but also reduces LDL-C by $10 \% .^{18}$ In the Coronary Drug Project, niacin reduced CVD events and was more effective than the comparator fibrateclofibrate. However, in the recent AIM-HIGH and HPS2/THRIVE studies, niacin did not add to statin (and ezetimibe) therapy, resulting in no CVD benefit, but did lead to an increase in rates of new diabetes, gastrointestinal side-effects and infections.

Individuals with inherited ultra-high HDL-C levels exist and as a result they have very low LDL-C levels. Some have mutations in cholesterol ester transfer protein (CETP). Epidemiological studies of migrants with CETP deficiency suggest a small CVD benefit of their impaired CETP function and raised HDL-C levels. ${ }^{19}$ Oral compounds that inhibit CETP have been developed. ${ }^{8}$ The first, torcetrapib, was highly effective in raising HDL-C by $100-150 \%$ but increased CVD events in the ILLUMINATE trial. This has been ascribed to its off-target actions in increasing aldosterone, corticosterone and endothelin-1 levels, leading to an average $5 \mathrm{mmHg}$ rise in blood pressure in the treatment arm of the trial. ${ }^{20}$ The second, the CETP inhibitor dalcetrapib, was designed to modulate and not completely inhibit CETP activity. Excess CETP activity is a feature of the process resulting in the formation of the small dense atherogenic LDL particles seen in type 2 diabetes. CETP is actually involved in modulating/converting particles containing apolipoprotein B or A-1, as well as the exchange shuttle of cholesterol for triglycerides between the two particle series. In the Dal-OUTCOMES study, dalcetrapib showed no benefit on CVD events in an ACS population. ${ }^{21}$ Two drugs anacetrapib and evacetrapib - remain in development. Both raise HDL-C by $80-120 \%$ and reduce LDL-C by $30-40 \%$. Neither raises blood pressure. Outcome studies are underway with both compounds in populations with chronic CVD 
(anacetrapib; HPS3/REVEAL) and acute coronary syndromes (evacetrapib; ACCELERATE). Results are expected in 2016-17. Ironically, even if the effects of CETP inhibitors on HDL-C prove irrelevant, these drugs may reduce CVD events simply as a by-product of the action to reduce LDL-C.

Controversy exists about whether the cholesterol content/ transport ability of HDL particles is actually the best measure of their function. Many of the multiplicity of apolipoproteins associated with HDL particles have anti-inflammatory actions. Irrespective of the exact function of HDL, infusions of HDL particles in animal models reduce atherosclerosis and in one proof-of-concept trial in man using an apolipoprotein- $\mathrm{A} 1_{\text {Milano, }}$ a hyperfunctional HDL particle showed a large decrease in intravascular ultrasound atheroma volume after only five infusions. ${ }^{22}$ Unfortunately it has to date proved impossible to synthesise these particles on a large scale.

\section{Interventions on triglycerides}

Triglycerides are a controversial risk factor for CVD. In many cases high triglycerides are simply the mirror of low HDL-C levels. Epidemiological studies suggest they may add to CVD risk above HDL-C, but the results are not consistent. Two existing classes of lipid-lowering drugs affect triglyceride levels. Fibrates (peroxisomal proliferator activating receptor-alpha [PPAR- $\alpha]$ agonists) reduce triglycerides and change small dense atherogenic LDL particles to large buoyant easily cleared ones. They can also raise HDL-C through increasing synthesis of apoA-1, and some reduce LDL-C. They have a modest benefit in reducing CVD events in meta-analyses of the trials over the last 50 years and may have additional benefits on microvascular disease in diabetes. ${ }^{23,24}$ The related PPAR- $\gamma$ agonists (glitazones and thiazolidinediones) have proved controversial. They reduce glucose levels and some have lipid benefits that resemble the actions of fibrates. Pioglitazone reduced CVD events in the PROACTIV trial but rosiglitazone may have increased CVD events in a meta-analysis of trials. Combined PPAR- $\alpha / \gamma$ agonists potentially offered beneficial effects on lipids and glycaemia ${ }^{8}$ but have proved either toxic or ineffective in reducing CVD events, as was the case with aleglitazar in the ALECARDIO study. ${ }^{25}$ Novel combined PPAR agonists have sought to combine PPAR$\alpha / \delta$ activity to maintain the lipid benefits and reduce musclebased insulin resistance. ${ }^{8}$ There is a need for CVD event trials in patients with residual high triglycerides after statin therapy and this may be where these agents are investigated.

Omega-3 fatty acids (docosahoexaenoic acid and eicosapentaenoic acid) at high doses reduce triglycerides in a dose-proportional manner and have anti-inflammatory effects at all doses. Low dose omega-3 fatty acids did reduce CVD events in the GISSI-P and JELIS trials but had no effect on CVD rates in the ORIGIN study in patients with diabetes/ impaired fasting glucose. Meta-analyses suggest they add little at low doses to placebo or to other treatments including statin therapies. ${ }^{26}$ Many novel preparations of omega- 3 fatty acids are in development but it remains to be seen whether they will be of any use in CVD prevention.

\section{Orphan drug therapies}

A number of orphan drug therapies have been developed for ultra-rare lipid disorders. Patients with homozygous FH
(HoFH) have vastly increased LDL-C and CVD rates and typically die by age 30 . They have a limited response to statins and many require treatment with lipid apheresis. Orphan drugs targeting the synthesis of apolipoprotein B particles have been developed. Mipomersen, an ASO, targets the apolipoprotein B mRNA, and reduces LDL-C by $25 \%$ in HoFH. It has problems with injection site reactions and hepatic steatosis but has an orphan drug license in the USA. ${ }^{27}$ Lomitapide, a microsomal transfer protein inhibitor, targets the lipidation of apolipoprotein B and can reduce LDL-C by $50 \%$ in HoFH. It causes profound hepatic steatosis and transaminase elevations. ${ }^{28}$ In addition, one case report suggests that lomitapide may be effective in the treatment of homozygous lipoprotein lipase deficiency (LPLD; familial chylomicronaemia). ${ }^{29}$ Lomitapide is licensed for HoFH in Europe. Alipogene tiparvovec is a gene therapy approach licensed in Europe which uses an adenoassociated virus vector to introduce LPL into muscle, reducing the incidence of pancreatitis in LPLD without obvious longterm effects on triglyceride levels. ${ }^{30}$

\section{Conclusions}

Many new lipid-lowering therapies are in development. The success of statins makes demonstrating the additional benefits of further lipid-lowering a difficult task. However the high efficacy on LDL-C and good safety profile of PCSK-9 inhibitors, and the high efficacy of CETP inhibitors in reducing LDL-C and raising HDL-C, means that if the clinical outcome trials are successful then these agents may be added to protocols for patients with the highest risks of developing recurrent or new onset CVD.

\section{Conflict of interest}

The authors have no conflicts of interest to declare. Professor Wierzbicki was chair of the NICE lipid modification guidelines (update) committee. The views in this article are his own and do not represent those of the committee.

\section{References}

1 Yusuf S, Hawken S, Ounpuu S et al. Effect of potentially modifiable risk factors associated with myocardial infarction in 52 countries (the INTERHEART study): case-control study. Lancet 2004;364:937-52.

2 The Scandinavian Simvastatin Survival Study (4S) investigators. Randomised trial of cholesterol lowering in 4444 patients with coronary heart disease: the Scandinavian Simvastatin Survival Study (4S). Lancet 1994;344:1383-9.

3 Baigent C, Blackwell L, Emberson J et al. Efficacy and safety of more intensive lowering of LDL cholesterol: a meta-analysis of data from 170,000 participants in 26 randomised trials. Lancet 2010;376:1670-81.

4 Stone NJ, Robinson J, Lichtenstein AH et al. 2013 ACC/AHA Guideline on the treatment of blood cholesterol to reduce atherosclerotic cardiovascular risk in adults: a report of the American College of Cardiology/American Heart Association Task Force on Practice Guidelines. Circulation 129(25 Suppl 2):S49-73.

5 National Clinical Guideline Centre. NICE guideline CG181 Lipid modification. 2014 27/07/2014. Available online at www.nice.org.uk/ guidance/CG181 [Accessed 1 August 2014].

6 Reiner Z, Catapano AL, De Backer G et al. ESC/EAS Guidelines for the management of dyslipidaemias: the Task Force for the management of dyslipidaemias of the European Society of Cardiology (ESC) and the European Atherosclerosis Society (EAS). Eur Heart J 2011;32:1769-818. 
7 Wierzbicki AS, Hardman TC, Viljoen A. New lipid-lowering drugs: an update. Int J Clin Pract 2012;66:270-80.

8 Hajhosseiny R, Sabir I, Khavandi K, Wierzbicki AS. The floods and ebbs in cholesterol lowering drug development: Prospects for the future. Clin Pharmacol Ther 2014;96:64-73.

9 Joy TR, Hegele RA. Narrative review: statin-related myopathy. Ann Intern Med 2009;150:858-68.

10 Meek C, Wierzbicki AS, Jewkes C et al. Daily and intermittent rosuvastatin $5 \mathrm{mg}$ therapy in statin intolerant patients: an observational study. Curr Med Res Opin 2012;28:371-8.

11 Wierzbicki AS. The ezetimibe Jonah: the trials and tribulations of an unlucky drug. Int J Clin Pract 2011;65:1207-8.

12 Wierzbicki AS, Hardman TC, Viljoen A. Inhibition of pro-protein convertase subtilisin kexin-9 (PCSK9) as a treatment for hyperlipidaemia. Expert Opin Investig Drugs 2012;21:667-76.

13 Raal FJ, Honarpour N, Blom DJ et al. Inhibition of PCSK9 with evolocumab in homozygous familial hypercholesterolaemia (TESLA Part B): a randomised, double-blind, placebo-controlled trial. Lancet 2014 , in press.

14 Blom DJ, Hala T, Bolognese M et al. A 52-week placebo-controlled tria of evolocumab in hyperlipidemia. N Engl J Med 2014;370:1809-19.

15 Fitzgerald K, Frank-Kamenetsky M, Shulga-Morskaya S et al. Effect of an RNA interference drug on the synthesis of proprotein convertase subtilisin/kexin type 9 (PCSK9) and the concentration of serum LDL cholesterol in healthy volunteers: a randomised, singleblind, placebo-controlled, phase 1 trial. Lancet 2014;383:60-8.

16 Birjmohun RS, Hutten BA, Kastelein JJ, Stroes ES. Efficacy and safety of high-density lipoprotein cholesterol-increasing compounds: a meta-analysis of randomized controlled trials. J Am Coll Cardiol 2005;45:185-97.

17 Robins SJ, Collins D, Wittes JT et al. Relation of gemfibrozil treatment and lipid levels with major coronary events: VA-HIT: a randomized controlled trial. JAMA 2001;285:1585-91.

18 Wierzbicki AS. Niacin: the only vitamin that reduces cardiovascular events. Int J Clin Pract 2011;65:379-85.

19 Chapman MJ, Le GW, Guerin M, Kontush A. Cholesteryl ester transfer protein: at the heart of the action of lipid-modulating therapy with statins, fibrates, niacin, and cholesteryl ester transfer protein inhibitors. Eur Heart J 2010;31:149-64.

20 Vergeer M, Stroes ES. The pharmacology and off-target effects of some cholesterol ester transfer protein inhibitors. Am J Cardiol 2009;104:32E-8E
21 Schwartz GG, Olsson AG, Abt M et al. Effects of dalcetrapib in patients with a recent acute coronary syndrome. $N$ Engl J Med 2012;367:2089-99.

22 Nissen SE, Tsunoda T, Tuzcu EM et al. Effect of recombinant ApoA-I Milano on coronary atherosclerosis in patients with acute coronary syndromes: a randomized controlled trial. JAMA 2003;290:2292-300.

23 Wierzbicki AS. Fibrates: no ACCORD on their use in the treatment of dyslipidaemia. Curr Opin Lipidol 2010;21:352-8.

24 Jun M, Foote C, Lv J et al. Effects of fibrates on cardiovascular outcomes: a systematic review and meta-analysis. Lancet 2010;375:1875-84

25 Lincoff AM, Tardif JC, Schwartz GG et al. Effect of Aleglitazar on cardiovascular outcomes after acute coronary syndrome in patients with type 2 diabetes mellitus: the AleCardio randomized clinical trial. JAMA 2014;311:1515-25.

26 Kotwal S, Jun M, Sullivan D et al. Omega 3 Fatty acids and cardiovascular outcomes: systematic review and meta-analysis. Circ Cardiovasc Qual Outcomes 2012;5:808-18.

27 Raal FJ, Santos RD, Blom DJ et al. Mipomersen, an apolipoprotein B synthesis inhibitor, for lowering of LDL cholesterol concentrations in patients with homozygous familial hypercholesterolaemia: a randomised, double-blind, placebo-controlled trial. Lancet 2010;375:998-1006.

28 Cuchel M, Meagher EA, du Toit Theron $\mathrm{H}$ et al. Efficacy and safety of a microsomal triglyceride transfer protein inhibitor in patients with homozygous familial hypercholesterolaemia: a single-arm, open-label, phase 3 study. Lancet 2013;381:40-6.

29 Sacks FM, Stanesa M, Hegele RA. Severe hypertriglyceridemia with pancreatitis: thirteen years' treatment with lomitapide. JAMA Int Med 2014;174:443-7.

30 Gaudet D, Methot J, Dery S et al. Efficacy and long-term safety of alipogene tiparvovec (AAV1-LPLS447X) gene therapy for lipoprotein lipase deficiency: an open-label trial. Gene Ther 2013;20:361-9.

Address for correspondence: Prof AS Wierzbicki, Department of Metabolic Medicine/Chemical Pathology, Guy's and St Thomas' Hospitals, London SE1 7EH, UK. Email: anthony.wierzbicki@kcl.ac.uk 